\title{
THE DENSITY OF SILICON TETRAFLUORIDE
}

BY ALBERT F. O. GERMANN AND HAROLD S. BOOTH

Among the gases whose physical constants have been little studied, the fluoride gases are perhaps pre-eminent. The reason for this seems to be the indication by most experimenters that these gases slowly attack glass at room temperature, ${ }^{1}$ giving rise to various by-products which would vitiate results; furthermore, the purification of these gases presents an unusually difficult problem. In recent years, however, the opinion has gained ground that many reactions will not proceed in the absence of traces of moisture. So, for example, perfectly dry nitric oxide gas, as well as nitrogen peroxide, when left in contact with dry mercury at room temperature, leaves the surface of the mercury unattacked; the merest trace of moisture, however, suffices to tarnish the surface. We decided, therefore, that the action of the fluoride gases on glass might depend upon the presence of traces of moisture.

Preliminary work on the subject, using silicon tetrafluoride as the experimental gas, both on account of the ease with which it may be prepared and because of its high fluorine content, demonstrated the fact that at least this fluoride, when perfectly dry, attacked neither glass nor mercury, even after five months of contact.

With this assurance, we decided to undertake the accurate determination of the weight of the normal liter of silicon tetrafluoride, and eventually of other physical constants of the gas, as the opportunity offered. This determination seemed all the more important because all but one of the published values were made before the modern technique in the measurement of gas densities had been developed. And it is evident that any effort to arrive at a satisfactory value for the atomic weight of fluorine by physico-chemical methods must end in failure until modern methods are applied.

${ }^{1}$ See, for example, H. Rose: Pogg. Ann., 80, 406 (1850). 


\section{Historical}

There have been very few determinations of the density of silicon tetrafluoride. Four of these are of historical interest only. The earliest is that of $\mathrm{J}$. Davy, ${ }^{1}$ who found its specific gravity; compared to air, to be 3.5735 . In 1827 Dumas $^{2}$ found a value of 3.6. Dalton found a value compared to air of 4.17 , which is apparently much too high. Truchot, ${ }^{3}$ in 1884 , following the method of Chancel, ${ }^{4}$ found for the value of the density of silicon tetrafluoride compared to air, 3.6. These determinations were made by crude methods, no adequate means having been used to purify the gas or to avoid the absence of air or of hydrogen fluoride.

There is only one modern determination of the density of silicon tetrafluoride, that of Jaquerod and Tourpaian, ${ }^{5}$ in I913. These authors used the principle of Archimedes, that of a light glass globe floating in the gas whose density is to be determined, and checked their results by the globe method. They found the value

$I_{N}($ silicon tetrafluoride $)=4.693 \mathrm{~g}$,

a value which according to the same authors ${ }^{6}$ gives for the atomic weight of fluorine, I9.09, which is unquestionably too high. The gas used was probably purer than any used before for a density determination, since, in addition to the usual precautions for its preparation, the gas was condensed and purified by fractional sublimation. A list of these values is given in Table I. A careful search of the literature has failed to reveal any others.

TABLE I

\begin{tabular}{l|c|lc}
\hline J. Davy & I8I2 & $3.5735($ air $=\mathrm{I})$ \\
Dumas & I827 & 3.6 & $" 6$ \\
Dalton & - & 4.17 & " \\
Truchot & I884 & 3.6 & \\
Jaquerod and Tourpaian & I9I3 & $\mathrm{L}_{\mathrm{N}}=4.693$
\end{tabular}

1 Phil. Trans., 102, 352 (1812).

${ }^{2}$ Ann. Chim. Phys., (2) 33, 370 (1826); Pogg. Ann., 9, 418 (1827).

${ }^{3}$ Comptes rendus, 98,821 (I 884 ).

${ }^{4}$ Ibid., 94, 626 (I882).

5 Jour. Chim. phys., II, 3 (I913).

${ }^{6}$ Ibid., II, 269 (1913). 


\section{Preliminary Work}

Our first efforts were directed toward determining whether silicon tetrafluoride attacked glass. For this purpose we set up a generator and prepared the gas, passing it through a drying tube, $D_{1}$ (Fig. 3), consisting of alternate sections of glass wool and phosphorus pentoxide to remove hydrogen fluoride and the water resulting from its reaction with the glass wool. Then we solidified this gas with the aid of liquid air. We let it stand in contact with the mercury of the manometer $\mathrm{M}_{1}$ over night and found no action on the glass or on the mercury in the morning; and this in spite of the fact that the silicon tetrafluoride had escaped by way of the manometer during the night. Thus encouraged we decided to make a preliminary determination of the density, using the apparatus shown in Fig. 3 with the exception that pressure manometers $\mathrm{M}_{2}$ and $\mathrm{M}_{3}$ were only safety manometers of a capacity of from a vacuum to atmospheric pressure. We tried to purify the gas by distilling from the solidified silicon tetrafluoride in one distilling tube to the other tube, rejecting the first and last fractions; in fact, the method used by Jaquerod and Tourpaian. We repeated this distillation, or rather sublimation, five times, hoping to obtain a pure gas. We observed during the sublimation that the current of gas from the surface of the solid carried along particles of a solid gas which soon volatilized when removed from the neighborhood of the solidified silicon tetrafluoride; this we had every reason to believe was an impurity. We endeavored to avoid this by slowing down the rate of distillation but were not successful. Using the gas which we had attempted to purify in this manner, we made a determination of the density. The value thus found for the density was too low and we were therefore forced to the conclusion that this method of sublimation was not a satisfactory method of purification for silicon tetrafluoride.

It is a well known fact that the most satisfactory method of purifying a gas, aside from the chemical methods, is the fractional distillation of the liquefied gas. ${ }^{1}$ Moissan $^{2}$ in-

${ }^{1}$ See Germann: Jour. Phys. Chem., I9, 45 I (Igr5).

${ }^{2}$ Comptes rendus, I39, 7 I I (rgO4). 
dicates that solid silicon tetrafluoride changes to a liquid under a pressure of two atmospheres and a temperature of $-77^{\circ} \mathrm{C}$. Our next step was to modify the apparatus so as to be able to carry out the purification by distilling the liquefied gas. The simplest method at hand was to construct a pressure device such as that shown in Fig. 3. Here the wide tubes A have a volume three times that of the narrow tubes $B$ per unit length, and tubes $B$ are three times as high as tubes $A$, so that, when pressure forces the mercury in the wide tube to a point near the bottom, the mercury rises three times as far in tube $B$, thus enabling us to work with a range of pressures from a vacuum to several atmospheres.

\section{Method}

The globe method essentially as described in detail by Germann ${ }^{1}$ was chosen for its rapidity and accuracy and was carefully followed out with the exception that three globes and only one barometer were used.

This method in our present state of knowledge eliminates practically all sources of error or reduces them to a minimum. For example, the use of several flasks eliminates gross errors in calibration and in weighing, and the possibility of error due to condensation of the gas upon the inner surface of the globe when the gas is admitted is materially reduced by using flasks of different sizes.

\section{Description of the Apparatus}

The apparatus used was of course constructed entirely of glass. In no place was any rubber tubing or foreign substance allowed to come in contact with the silicon tetrafluoride except in the stopcocks and at the ground flat joints $\mathrm{J}$ by which the globes were attached to the apparatus. Here a special rubber-vaseline grease was used which our experience shows is practically unaffected by silicon tetrafluoride. The stopcocks on the apparatus were all of the capillary variety, especially made for vactum work.

1 Jour. Phys. Chem., I9, 437 (I915). 
The barometer used was the type described by Germann ${ }^{1}$ with a glass scale made by the Sociéte Genevoise pour la Construction des Instruments de Physique. The barometer was carefully cleaned by filling with chromic acid and allowing it to stand over night. It was then rinsed and filled with strong nitric acid containing a few drops of alcohol and allowed to stand for several hours and then washed with distilled water. It was then placed in position, and air, dried by sulphuric acid and phosphorus pentoxide, was passed through the apparatus continuously for one week to remove all adsorbed moisture. After being allowed to stand evacuated for several days, it was filled with mercury without heating. This mercury was purified by first agitating with ro\% nitric acid by means of a suction pump, next passing through a mercury tower containing the same reagent, and finally distilled in a vacuum in an all-glass apparatus.

The temperature of the room was so constant and the time of reading was so short that it was found unnecessary to surround the barometer with an air bath. Three carefully calibrated thermometers were mounted beside the glass scale, one near the upper mercury surface, one near the center and one at the bottom. The barometer was read with the aid of a cathetometer, an electric light immersed in a bath of water arranged on a pulley behind the barometer case providing illumination. The corrected barometer reading was obtained by applying the temperature ${ }^{2}$ and gravity ${ }^{3}$ corrections. The residual pressures in the globes were measured with a modified McLeod vacuometer which had a ratio of one actual millimeter to $19.16 \mathrm{~mm}$ as read on the scale. Vacuum was obtained by a water suction pump and the mercury pump. The globes in which the gas was weighed were round bottom flasks selected from the stock of distilling flasks; these were drawn out at the neck and glass stopcocks with

${ }^{1}$ Jour. Am. Chem. Soc., 36, 2456 (1914).

${ }^{2}$ Landolt-Börnstein, page I8 (I9I2).

${ }^{3}$ See page 86 .

${ }^{4}$ Germann and Cardoso: Jour. Chim. phys., Io, 406 (I9I 2 ). 
flat joints sealed on (see $J$ in Fig. 3). Flasks from the same stock were chosen corresponding in air displacement to the density globes and filled with distilled water until they weighed two grams more than the corresponding glass-cocked flask. They were then sealed off in a crook to be used as counterpoises. The density globes were calibrated according to the method outlined by Germann. ${ }^{1}$ This method is simply evacuating and weighing, filling the globes with air-free distilled water at zero and weighing, and then emptying, evacuating and weighing. The difference between the weight filled and the average of the weights evacuated was the weight of water contained at the temperature of melting ice.

To find the volume at zero, the following corrections had to be applied:

I. Correction for the buoyancy of the air on the weights used. The average weight of a cubic centimeter of air in Cleveland is $0.00117 \mathrm{~g}$, and therefore the correction amounted to $\frac{\mathrm{W}}{8.5}$ $X 0.001 \mathrm{I} 7$, where $\mathrm{W}$ is the weight of water and 8.5 is the density of brass; the correction is negative.

2. Correction for volume of water at zero; if the density: of water at zero is taken as 0.999868 , the correction is $\mathrm{W} \times$ $0.00013^{2}$ and is positive.

3. Correction for latitude and altitude. The value of the gravity constant at Cleveland may be theoretically determined by substitution in the following formula:

$$
\mathrm{G}_{\circ}=\frac{\mathrm{I}+\frac{2 h}{\mathrm{R}}}{\mathrm{I}-\delta \cos 2 \phi}
$$

where $h$ is the altitude above sea level which at the well of our barometer was 219.5 meters, where $R$ is the average radius of the earth; 6367382 meters, and where $\phi$ is the latitude at the point of / observation, here, $4 \mathrm{I}^{\circ} 3 \mathrm{O}^{\prime} \mathrm{1} 5^{\prime \prime}$, and where $\delta$ is a constant which is given as 0.0025935 by Lasch. ${ }^{2}$

${ }^{3}$ Jour. Phys. Chem., 19, 459 (I915).

${ }^{2}$ Pogg. Ann. Erg., 3, 32 I (1853). 
Substituting these values in this equation we get the value of 1.0003847 . The ratio of the gravity constant at sea level, $\mathrm{g}_{n}$, to the gravity constant, $\mathrm{g}_{m}$, as determined experimentally in our physics laboratory, is

$$
\begin{aligned}
\mathrm{G}_{0}=\frac{\mathrm{g}_{n}}{g_{m}}=\frac{980.6 \mathrm{I} 6}{980.240} & =\mathrm{I} .0003835 \\
\text { Theoretical Value } & =\mathrm{I} .0003847 \\
\text { Experimental Value } & =\underline{\mathrm{r} .0003835} \\
\text { With a difference of only } & 0.00000 \mathrm{I} 2
\end{aligned}
$$

The values were averaged and rounded off to $G_{0}=1.000384$, which is the most probable value of the gravity constant at the Morley Laboratory.

The algebraic sum, $\Sigma$, of these corrections becomes $\Sigma$ $=0.000384 \mathrm{~W}+0.000132 \mathrm{~W}-0.000138 \mathrm{~W}$; therefore $\mathrm{W}+$ $0.000378 \mathrm{~W}=$ true volume of globes.

\begin{tabular}{|c|c|c|c|}
\hline & Volume & Correction & Corrected volume \\
\hline Globe $\mathrm{E}$ & $332.487 \mathrm{~g}$ water & 0.126 & $332.6 \mathrm{I} \mathrm{cc} \mathrm{Booth}$ \\
\hline Globe F & $573.435 \mathrm{~g}$ water & 0.216 & 573.65 cc Booth \\
\hline Globe D & $203.658 \mathrm{~g}$ water & 0.077 & 203.765 cc Germann \\
\hline Globe $A$ & $63 \mathrm{I} .17 \mathrm{~g}$ water & 0.238 & $63 \mathrm{I} .4 \mathrm{I}$ cc Germann \\
\hline
\end{tabular}

The following table gives the results of several concordant measurements on each globe:

TABLE II

An important source of systematic error that was neglected until Rayleigh called attention to it in $r 888^{1}$ is the contraction of the globe when it is evacuated, causing it to displace less air than before and hence apparently to weigh more. To correct this error it is necessary to find the amount of the contraction. Fig. I shows the globe enclosed in a stout inverted bell jar supplied with a flat plate glass top which could be clamped on tightly by thumb screws. This flat plate had an inch hole provided with a one-holed rubber stopper slit so as to permit putting it on the neck of the flask.

1 Proc, Roy. Soc., 43, 36I-2 (r888); Chem. News, 57, 74 (I 888). 
The calibrated capillary tube was fitted into the lid by grinding it into place in a hole through the plate. The bell

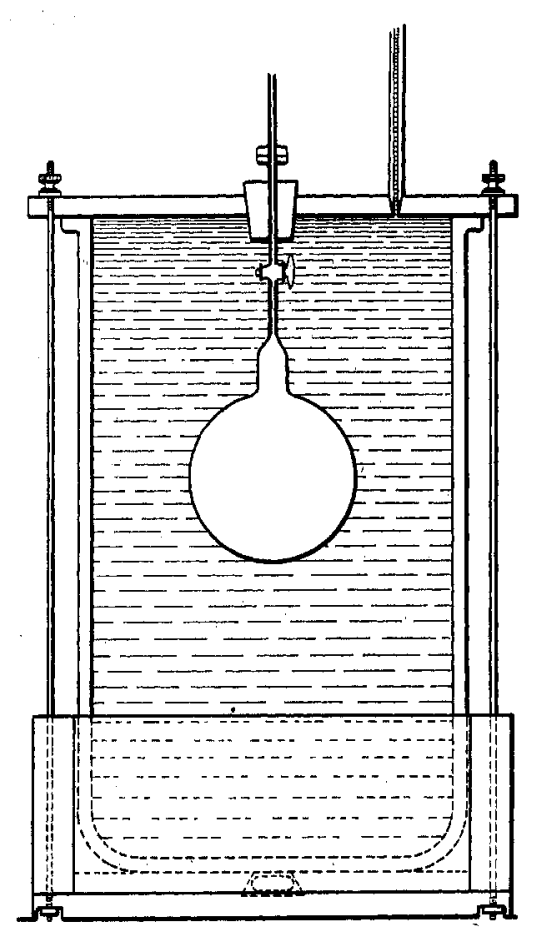

Fig. I jar was filled with water, the rim greased with rubber-grease and the flat plate clamped on so that the water stood at some point in the capillary; the globe was evacuated; the level of the water in the capillary tube noted; then air was allowed to fill the globe rapidly and the level of the water again noted. The volume of the capillary between the two points noted was the amount of contraction of the globe. The average of several determinations was taken as the final value. Results are included in Table III.

The weights used in this work were gold plated brass weights, supplied by the Scientific Materials Co. and were carefully calibrated against a standard ten gram weight (Reichsanstalt) according to the Kohlrausch method.

TABLE III

\begin{tabular}{|c|c|c|c|}
\hline & $\begin{array}{l}\text { Gross weight } \\
\text { of flask }\end{array}$ & Contraction $\mathrm{cc}$ & Air displaced per liter \\
\hline Globe D & 65 grams & $0.008 \mathrm{cc}$ & $0.000047 \mathrm{~g}$ Germann \\
\hline Globe $\overline{\mathrm{E}}$ & I I 3 grams & $0.0232 \mathrm{cc}$ & $0.00008 \mathrm{~g}$ Booth \\
\hline Globe $F$ & I 39 grams & $0.0495 \mathrm{cc}$ & $0.00010 \mathrm{~g}$ Booth \\
\hline Globe A & I 3 grams & $0.040 \mathrm{cc}$ & $0,000074 \mathrm{~g}$ Germann \\
\hline
\end{tabular}

An ordinary chemical balance was employed. To increase the accuracy the pans were removed and light aluminium 
trays were suspended close under the knife edges by means of fine platinum wire. These trays were designed to receive the weights and leave the lower part of the balance case free to receive the globes and their counterpoises. The balance case itself was surrounded by a much larger glass case with doors opening from the front. Across the top of this outer case was stretched a nickel wire from which the globes with their counterpoises were hung preparatory to weighing.

For the preparation and purification of the silicon tetrafluoride used, special forms of apparatus were e m ployed. The generator $\mathrm{G}$ (see Figs. 2 and 3) was made of a large thick-walled round-bottomed distilling flask, the neck of which bore two tubes, the delivery tube $\mathrm{D}$ and tube $\mathrm{H}$ for admitting the sulphuric acid. The reservoir $\mathrm{R}$ containing the conc. sulphuric acid was made of a large calcium chloride tube. To control the amount of sulphuric acid being admitted to the flask, a needle valve, consisting of a large glass stirring rod, $S$, ground in place in the small end of the tube and held

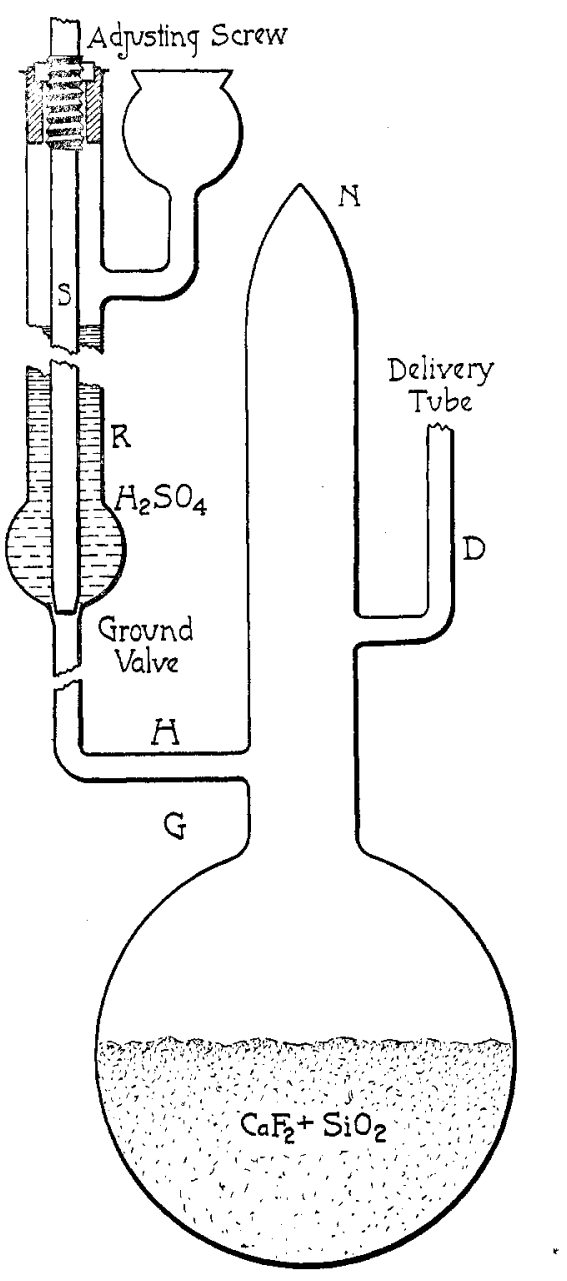

Fig. 2 in place at the top, was used. The adjustment was controlled by means of a threaded collar slipped over this rod and held in place by sealing wax; this threaded collar engaged threads of a brass nut fastened on a cork which fitted snugly in the 
9o Albert F. O. Germann and Harold S. Booth

upper end. When the needle valve was turned down into place, there was no difficulty in getting a perfect vacuum in

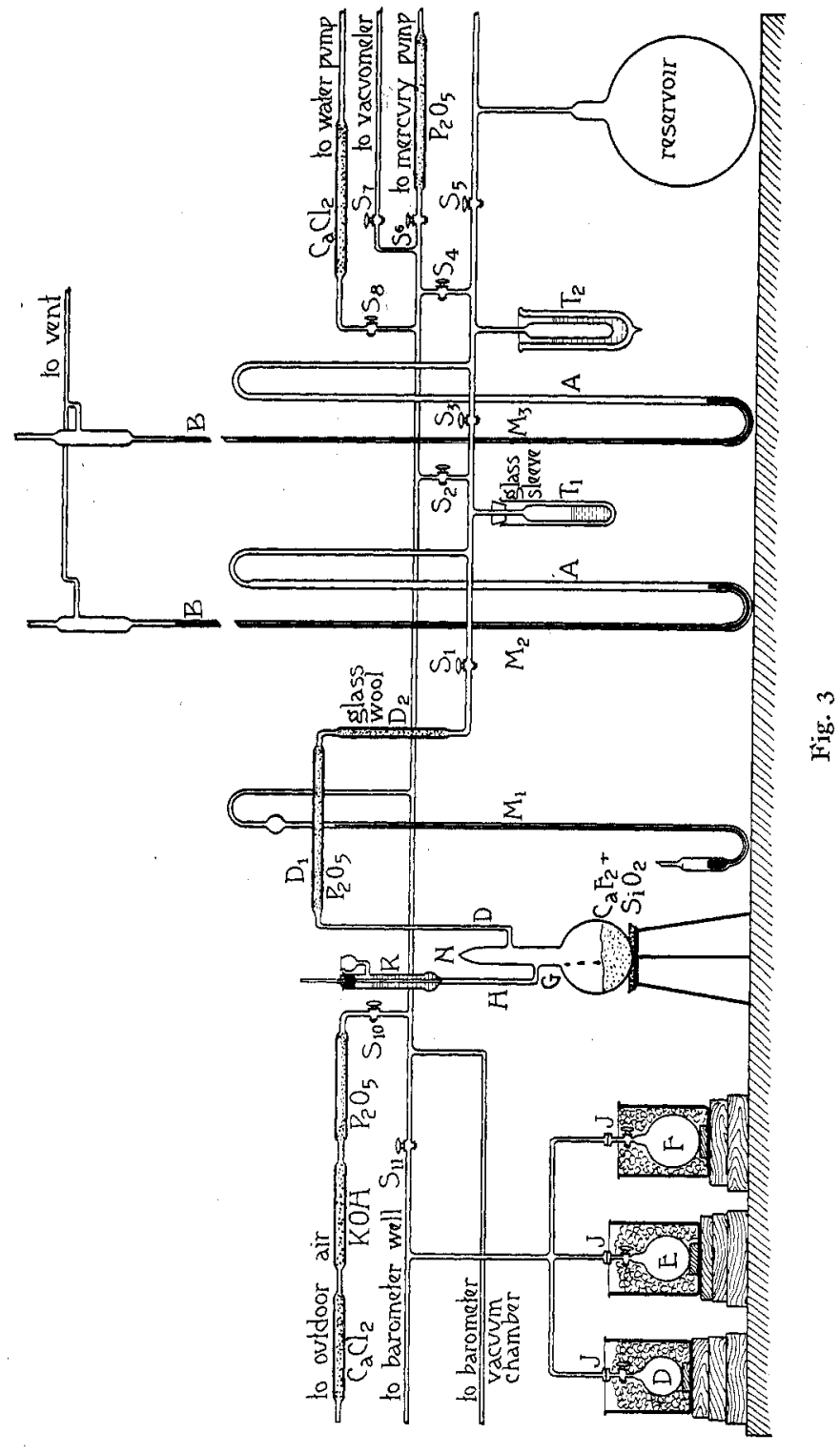


the flask. ${ }^{1}$ No heat was applied in generating gas, the sand bath being used merely as a support for the flask, and for safety in case of accident.

The gas generated in the flask passed from the delivery tube through a drying tube, $D_{1}$, containing seven alternate plugs of glass wool and phosphorus pentoxide and then through tube $\mathrm{D}_{2}$ containing glass wool. In the first tube the plugs of glass wool reacted with any hydrofluoric acid generated, forming silicon tetrafluoride, the water from this reaction being absorbed by the phosphorus pentoxide. No action was observed in this tube farther than the third plug.

From this point the gas passed into the apparatus designed for purification by physical methods. Through the stopcock $\mathrm{S}_{1}$ gas entered directly into the liquefaction bulbs $T_{1}$ and $T_{2}$ separated by stopcock $S_{3}$, each supplied with a manometer, $\mathrm{M}_{2}$ and $\mathrm{M}_{3}$.

In distilling from one bulb to the other, a glass sleeve was placed around the bulb from which the gas was distilling in order to prevent superheating. To the distilling apparatus a reservoir of about fifteen or twenty liters capacity was connected through stopcock $S_{5}$. Each distilling bulb was also connected through a stopcock, $S_{2}$ and $S_{4}$, to a main line to which was connected by means of stopcocks a mercury pump, vacuometer, water suction pump and a safety manometer, $M_{1}$. To this main line, through a series of drying and absorption tubes carbon-dioxide-free and water-free air could be admitted through stopcock $\mathrm{S}_{10}$. Through stopcock $S_{11}$ gas was admitted to the globes and to the well of the barometer. During distillation stopcocks $S_{1}, S_{2}, S_{3}, S_{4}$ and $S_{5}$ were provided with brass clamps to withstand the pressure.

\section{Manipulation}

Before beginning the measurements the globes wereclamped in position and the entire apparatus was evacuated and

${ }^{1}$ Both these pieces of apparatus and the apparatus used for the determination of the contraction of the globes were home-made affairs, but answered their respective purposes as well as the imported apparatus. 
allowed to stand over night in contact with the phosphorus pentoxide tube of the mercury pump.

\section{Generation of Gas}

The flask $\mathrm{G}$ was half filled with a well ground mixture of one part calcium fluoride and two parts pure sea sand. Broken pieces of soda glass were scattered over the surface of this mixture to insure a large excess of silica. Then the neck of the flask was drawn out and sealed off at $N$ (Figs. 2 and 3 ). Sulphuric acid was placed in the reservoir R and the ground valve opened enough to wet it and then reseated tightly. The generating flask was evacuated and left to stand over night in contact with phosphorus pentoxide in $D_{1}$ (Fig. 3). The large reservoir was similarly evacuated as completely as possible and the whole system was left in contact with the phosphorus pentoxide of the mercury pump through $\mathrm{S}_{6}$. Next morning sulphuric acid was allowed to drip slowly upon the mixture in the generator, and the gas evolved condensed in $T_{1}$ by surrounding it with liquid air in a Dewar flask, as at $T_{2}$.

When a sufficient quantity of the solid silicon tetrafluoride had been condensed the supply of acid was shut off and when generation ceased, stopcock $\mathrm{S}_{1}$ was closed.

\section{Distillation and Purifieation}

All stopcocks communicating with the distilling tubes were then clamped. The liquid air was changed to surround $T_{2}$, while $T_{1}$ was wiped with alcohol and the glass sleeve slipped over it. (Fig. 3 shows this arrangement.) The pressure in $T_{1}$ was allowed to increase until nearly all the silicon tetrafluoride was liquefied. The fractionation of the liquid followed. The first and last fractions-no less than 200 or 300 cubic centimeters of gas in each case-were allowed to escape by opening, stopcock $S_{2}$ and pumping off with the water pump. The middle fraction was condensed in $T_{2}$. At least five such distillations were made before the gas was considered to be pure. During the last two distillations the 
gas distilled at a constant pressure and the purified liquid was perfectly limpid and colorless.

In the distillation of liquefied gases a criterion of the purity of the gas is given by the pressure exerted during the distillation. The pressure remains constant as long as the composition of the distillate does not change and the distillation proceeds quietly.

Stopcock $S_{5}$ was finally gradually opened and the silicon tetrafluoride distilled into the reservoir.

The difficulty attending the handling of silicon tetrafluoride is the constant necessity for watcbfulness against the presence of water vapor. To make sure that the apparatus was perfectly dry, the whole system before filling was left evacuated in contact with phosphorus pentoxide. The next day the apparatus, including the density globes, was rinsed three times with air, free from moisture and carbon dioxide, admitting the air through stopcock $S_{10}$ and pumping it out by means of the water and mercury pumps. Then the rinsing was continued using silicon tetrafluoride admitted from the large reservoir, until it was deemed that the apparatus was free from air. In this case, the evacuation was facilitated by the use of liquid air, condensing all the gas used for rinsing purposes in one of the condensing tubes, and evacuating from this reduced volume; it is evident that any non-condensable gases would be dragged into the condensing tube, along with the silicon tetrafluoride. The residual pressure of silicon tetrafluoride, by means of the vacuometer, and the temperature, were noted. The stopcocks of the globes and all stopcocks except $S_{11}$ were then closed. The connecting tubing was then rinsed several times with dry air admitted slowly through $S_{10}$. Stopcocks $S_{10}$ and $S_{11}$ were then closed and the globes removed. The rubber-grease was washed from the flat joints with ether and the globes and counterpoises were washed with distilled water and wiped dry with a clean cloth and then wiped bright with a piece of good quality of white silk which left no lint. The globes and counterpoises were suspended in the outer balance case and weighed the next 
morning. The weighings were carried out exactly as described by one of us. ${ }^{1}$ Globes $\mathrm{D}$ and $\mathrm{E}$ were allowed to swing on the knife edges ten minutes, Globes F and A, fifteen. Three sets of weighings were made and the average of the three weighings taken.

This represented the weight that had to be added to the globe plus the small weight of silicon tetrafluoride it contained at a pressure of $p$ millimeters, to cause equilibrium to be established with the counterpoise. It was necessary to correct, then, for the small amount of silicon tetrafluoride still in the globe; if $\mathrm{V}$ be the volume of the globe expressed in $\mathrm{cc}, p$ the pressure in millimeters read on the vacuometer, and $T$ the absolute temperature of the gas when the reading was made, the amount of the correction is given by the expression

$$
\frac{4.684 \times 273 \times \mathrm{V} \times p}{1000 \times 760 \times \mathrm{T}}=\frac{0.001682 \mathrm{Vp}}{\mathrm{T}} \text {. }
$$

This correction is positive and must be added to the weight found.

The globes were then transferred to the laboratory, where they were placed in position, and the pressure in each measured to be certain that no air had entered in the interval, care having been taken to evacuate and rinse with air and then silicon tetrafluoride several times the space between the stopcocks of the globes and $\mathrm{S}_{11}$.

The afternoon was devoted to the fractionation of silicon tetrafluoride from the large reservoir and filling the globes with it: A sufficient quantity for filling the globes was condensed in tube $T_{2}$ and refractionated as already described. The first and last fractions, no less than 200 or $300 \mathrm{cc}$ of gas in each case, were pumped off. The middle fraction was condensed in the second liquefaction tube. At least three such distillations were made before the gas was considered to be pure. Stopcocks connecting to the globes were then opened. By lowering the Dewar flask surrounding the distilling tube containing the liquid silicon tetrafluoride, the liquid gas was allowed to distil slowly into the globes. When the pressure

1 Jour. Phys. Chem., I9, 466 (1915). 
of the gaseous silicon tetrafluoride in the globes neared 760 $\mathrm{mm}$, the barometer was placed in communication with the apparatus and distillation stopped when the pressure was approximately $762 \mathrm{~mm}{ }^{1}$ Two pressure readings were made on the barometer with the aid of the cathetometer and the average of the readings taken. The thermometers accompanying the barometer were read as the pressure readings were made. The globe stopcocks were closed and then those leading to the barometer.

After the silicon tetrafluoride in the rest of the apparatus had been recondensed in the distilling tube, the system was thoroughly rinsed with dry air. This ended the operation of filling. The globes were removed from the ice, carefully washed, wiped dry and placed in the weighing case as before to remain over night. Three weighings were made the next day. The averages of these weighings were the weights of the globes filled with silicon tetrafluoride at the pressure $\mathrm{P}$ read on the barometer.

On the afternoon after the weighing, the globes were evacuated by condensing the gas in tube $T_{1}$ and allowing the gas to distil into the reservoir. This gas was thus constantly being used and purified by fractionation. The globes were then weighed exactly as already described. The averages of the corrected weights of the globes evacuated before and after filling were considered the real values of the weights of the evacuated globes. Then the difference between the weight of the filled globe and that of the empty globe gave the weight of the gas it contained at zero degrees centigrade and $\mathrm{H} \mathrm{mm}$ pressure. The calculation of the gross weight of the liter followed by the application of this formula:

$$
L_{\text {gross }}=\frac{760 \mathrm{~W}}{\mathrm{HV}}
$$

Where $W$ is the weight of the gas found and $I_{\text {gross }}$ the uncorrected weight of the liter of gas. The corrections to be applied were as follows:

${ }^{1}$ Application of barometer correction brought this value back to about $760 \mathrm{~mm}$. 
I. A positive correction for the contraction of the globes during evacuation. Since this contraction involves an apparent increase in the weight of the globe when it is evacuated, due to the smaller volume of air displaced by it, the value of the correction $\mathrm{C}_{c}$ is evidently given by the additional weight of air that would be displaced if the globes were not evacuated. This weight on the basis of a liter, assuming the average weight of a cubic centimeter of air in Cleveland to be $0.00117 \mathrm{~g}$, and expressing the amount of the contraction in cubic centimeters by $\mathrm{C}$, is given by

$$
\mathrm{C}_{c}=\frac{1000 \times 0.00117 \times \mathrm{C}}{\mathrm{V}}=\frac{1.17 \mathrm{C}}{\mathrm{V}} .
$$

Substituting the values for the contraction found for the globes used in this formula, we obtained the positive corrections set down in Table III.

2. Correction for the buoyancy of air on the weights. Since only those weights come into consideration that are used to replace the silicon tetrafluoride when the globes are empty, the gross weights employed may be neglected and the calculation made on the basis of a liter, $4.684 \mathrm{~g}$. The weight of air displaced, $\mathrm{R}_{v}$, using as the density of brass 8.5 and assuming that all weights were made of brass ${ }^{1}$ is given by

a negative correction.

$$
\mathrm{R}_{v}=\frac{4.684 \times 0.001 \mathrm{I7}}{8.5}=0.0006445 \mathrm{~g}
$$

3. Positive correction for the altitude or reduction to sea level and $45^{\circ} \mathrm{N}$ latitude. This correction, $\mathrm{G}$ (see page 86 this article) is made as follows:

$\mathrm{G}=\mathrm{L}_{\mathrm{gross}}(\mathrm{r} .000384-\mathrm{r} .00)=4.684 \times 0.000384=0.00180$

4. Correction for the compressibility of silicon tetrafluoride. This correction was very small because the pressure at which the globes were closed before weighing, was always as nearly as possible equal to $760 \mathrm{~mm}$, the average of the pressures used being $760.4 \mathrm{I} \mathrm{mm}$. The value of the coefficient of compressibility for silicon tetrafluoride derived from critical data is, according to Jaquerod and Tourpaian, ${ }^{2}$

${ }^{1}$ See Gray and Burt: Jour. Chem. Soc., 95, I636 (Ig09).

${ }^{2}$ Jour. Chim. phys., II, 3 (I9I3). 


$$
\begin{aligned}
A_{0}^{\prime} & =0.005244 \\
\text { and } A & =0.00524 \times \frac{760-760.4 \mathrm{I}}{760} \mathrm{~L}=-0.00013 \mathrm{~g} .
\end{aligned}
$$

Applying these corrections to the gross weight of the liter of gas we have the expression

$$
\mathrm{L}_{\mathrm{N}}=\mathrm{L}_{\mathrm{gross}}+\mathrm{C}_{c}-\mathrm{R}_{v}+\mathrm{G}+\mathrm{A} .
$$

In practice the correction for the contraction of the globes during evacuation, $\mathrm{C}_{c}$, was applied to the individual results, since the correction varied from globe to globe; the other corrections were applied to the average obtained.

\section{Results}

The results obtained for the density of silicon tetrafluoride purified by fractional distillation under pressure as described are tabulated in Table IV. Globe F was broken when being taken from the ice in the second measurement and thereafter Globe $\mathrm{A}$ took its place. The barometric readings in the third column are the corrected readings. The gross weight in the fourth column is the weight of the silicon tetrafluoride contained in the globe at the time of measurement corrected for the inaccuracies of the weights. The value $L_{\text {gross }}$ is obtained by application of Boyle's law, while in the next column the weight of the gross liter has been

\begin{tabular}{|c|c|c|c|c|c|}
\hline $\begin{array}{l}\text { Measure- } \\
\text { ment }\end{array}$ & Globe & $\begin{array}{c}\text { Corrected } \\
\text { barometer } \\
\text { mm }\end{array}$ & $\begin{array}{c}\text { Gross weight } \\
\mathrm{g}\end{array}$ & $\begin{array}{l}\text { Lgross } \\
\quad \mathrm{g}\end{array}$ & $\underset{\mathrm{g}}{\operatorname{Lgross}}+\mathrm{C}_{c}$ \\
\hline I & $\mathrm{D}$ & $76 \mathrm{I} \cdot 42$ & 0.93755 & 4.59255 & 4.59260 \\
\hline I & $\mathrm{E}$ & $76 \mathrm{I} \cdot 42$ & I. 53064 & $4 \cdot 59332$ & $4 \cdot 59340$ \\
\hline I & F & $76 \mathrm{I} \cdot 42$ & 2.63884 & $4.592 \times 3$ & 4.59223 \\
\hline II & $\mathrm{D}$ & $759 \cdot 7^{6}$ & $0.9440 \mathrm{r}$ & 4.63430 & 4.63435 \\
\hline II & $E$ & 759.76 & I. 5403 I & 4.63244 & 4.63252 \\
\hline III & $\mathrm{D}$ & $761 \cdot 52$ & 0.95586 & 4.68 I 62 & 4.68 I 67 \\
\hline III & $\mathrm{E}$ & 76 I. 52 & I. 56036 & 4.68 I 89 & $4.68 I 97$ \\
\hline III & $\mathrm{A}$ & $76 \mathrm{I} \cdot 5^{2}$ & 2.96280 & 4.68285 & 4.68292 \\
\hline IV & $\mathrm{D}$ & $7.59 \cdot 30$ & 0.95343 & 4.68338 & 4.68343 \\
\hline IV & $E$ & $759 \cdot 3^{\circ}$ & I. 55745 & 4.68682 & 4.68690 \\
\hline IV & A & $759 \cdot 3^{\circ}$ & 2.95274 & 4.68075 & 4.68082 \\
\hline
\end{tabular}
corrected for the contraction of the globes (see Table III).

TABLE IV 
It will be remembered that we were continually re-using and purifying the same gas by fractionally distilling and storing in the reservoir. It is apparent in Table IV, which records the results of all measurements made by us, that the density was progressively rising (corresponding to greater purity) until the third set of measurements. In sets III and IV the results were concordant. This was considered an index to the absolute purity of the gas. ${ }^{1}$ Although at each fractionation the liquefied gas seemed perfectly limpid and colorless, yet we cannot consider that fact a sufficient criterion and have used only sets III and IV in the calculation of the final result.

The average of the values of the last two sets of measurements is $\mathrm{L}_{\text {gross }}+\mathrm{C}_{c}=4.68295$. The weight of the normal liter becomes, using the values already found for $\mathrm{R}_{v}, \mathrm{G}$ and $\mathrm{A}$ :

$$
\begin{gathered}
\mathrm{L}_{\mathrm{N}}=\left(\mathrm{L}_{\mathrm{gross}}+\mathrm{C}_{c}\right)-\mathrm{R}_{v}+\mathrm{G}+\mathrm{A}=4.68397 \mathrm{~g} \\
\text { Discussion of Results }
\end{gathered}
$$

\section{Discussion of Results}

As has been mentioned, the only value for the density of silicon tetrafluoride which may be classed as modern that to our knowledge has been published up to the present time, is that of Jaquerod and Tourpaian, ${ }^{2}$ who found

$$
\mathrm{L}_{\mathrm{N}}=4.693 \mathrm{~g} \text {. }
$$

This value differs from the value found in this research by two parts in one thousand, a difference which is too great to be attributed merely to experimental error. The methods used in both researches are standard methods, which have been used successfully by the same experimenters on other gases. $^{3}$

${ }^{1}$ It is also possible that equilibrium had not been established between the glass of the globes and the gas.

2 Loc, cit.

3 See the authors: Western Reserve University Bulletin, Vol. I9, No. 8, page 45 (I9I6), on "The Density of Air in Cleveland.;" here the same apparatus was used, and the value found for air,

$$
\mathrm{I}_{\mathrm{N}} \text { air }=\mathrm{I} .29273 \mathrm{~g}
$$

coincides with the average of results of other investigators. Also A. F. O. Germann, "The Density of Oxygen," Jour. Phys. Chem., 19, 437 (1915); and "The 
Hence it would seem that the error must be found in another direction. The purity of the gas employed may next be considered. We have already pointed out that the gas we used was used over and over, being subjected to renewed purification after each set of measurements. The results show that by this procedure some impurity, lighter than the gas in question, was progressively eliminated, until in the third set of measurements the purification seems to have been complete, inasmuch as no further change in density was found. It may be recalled at this point too, that our first efforts at purification, by distillation under diminished pressure, were entirely fruitless so far as the preparation of a pure gas is concerned, the values so found having all been too low. In the preparation of the gas, it will be recalled, we used no heat, but allowed the reaction to go on at room temperature, the heat of reaction being rather low, and in the presence of a large excess of finely divided silica and of glass wool. This was amply sufficient to arrest all hydrogen fluoride, as at the end of the measurements no part of the apparatus showed even the slightest etching. Jaquerod and Tourpaian, on the other hand, carried out the preparation of the gas by heating the reacting materials, and subsequently passing the gas over glass wool at $100^{\circ} \mathrm{C}$. The preliminary work of these authors brought out the fact that when the gas was passed over red hot glass wool, the results were too high, which caused them to suspect the formation of a sesquifluoride of silicon. ${ }^{1}$ Hence their adoption of the lower temperature to remove hydrogen fluoride. They, however, gave no evidence which would indicate that the formation of sesquifluoride did not take place

Density of Air in Geneva," Jour. Chim. phys., I2, I03 (1914), who found the following values by the globe method:

$\mathrm{L}_{\mathrm{N}}$ oxygen $=\mathrm{I} .42905 \mathrm{~g}$

$L_{\mathrm{N}}$ air $=\mathrm{I} .2930 \mathrm{~g}$

Jaquerod and Tourpaian: Arch. Sci. phys. nat., (4) 3I, 20 (I9II), who found, applying the principle of Archimedes,

$\mathrm{L}_{\mathrm{N}}$ oxygen $=\mathrm{I} .4290 \mathrm{~g}$.

1 Troost and Hautefeuille: Ann. Chim. Phys., (5) 7, 464 (1876). 
at $100^{\circ} \mathrm{C}$. Assuming the formula of the sesquifluoride to be $\mathrm{Si}_{4} \mathrm{~F}_{6}$, by analogy with silicon sesquichloride, a simple calculation will show that only o.I6 percent by weight of this impurity would suffice to raise the density of silicon tetrafluoride by two parts in one thousand. Moreover, the method of fractional distillation used by Jaquerod and Tourpaian is, to say the least, unsatisfactory, inasmuch as the distillation, or rather sublimation, proceeds only on the surface of the solid, where less volatile impurities, having once collected on the surface, may easily be carried along by the current of volatilizing gas-a circumstance, besides, which was actually observed in the course of our preliminary experiments. This is, of course, entirely prevented by the liquefaction of the gas by the application of pressure.

The theoretical value of the clensity of the normal liter of silicon tetrafluoride, considering the molecular weight to be I04.3, is lower than either of the values discussed above. ${ }^{1}$ Our value, $4.68397 \mathrm{~g}$, differs from it, therefore, much less than the value found by Jaquerod and Tourpaian. As the fifth decimal place has little significance, this may be rounded off to $4.684 \mathrm{O} \mathrm{g}$.

\section{Conelusion}

Considering the care taken in purifying the gas used in these measurements, and the precautions taken generally throughout this series of measurements, we believe that the value for the density of silicon tetrafluoride found above more nearly expresses the true density of the gas than any yet published. We therefore propose as the weight of the normal liter of silicon tetrafluoride,

$$
\mathrm{L}_{\mathrm{N}}=4.6840 \mathrm{~g} \text {. }
$$

In conclusion, we wish to express our gratitude to the Linde Air Products Co., of Cleveland, who supplied the liquid air for this research, and but for whose coöperation this work could not have been carried out.

The Morley Chemical Laboratory

Western Reserve University

Cleveland, Ohio

${ }^{1}$ A study of this subject will be undertaken in a separate paper. 